\title{
Weighted composition operators between weak spaces of vector-valued analytic functions
}

\section{Hassanlou, Mostafa}

2017

Hassanlou , M , Laitila , J A \& Vaezi , H 2017 , ' Weighted composition operators between

weak spaces of vector-valued analytic functions ' , Analysis , vol. 37 , no. 1 , pp. 39-45 . https://doi.org/10.1515/anly-

http://hdl.handle.net/10138/231796

https://doi.org/10.1515/anly-2015-0024

publishedVersion

Downloaded from Helda, University of Helsinki institutional repository.

This is an electronic reprint of the original article.

This reprint may differ from the original in pagination and typographic detail.

Please cite the original version. 


\title{
Research Article
}

Mostafa Hassanlou, Jussi Laitila* and Hamid Vaezi

\section{Weighted composition operators between weak spaces of vector-valued analytic functions}

DOI: $10.1515 /$ anly-2015-0024

Received June 11, 2015; accepted September 6, 2016

\begin{abstract}
We consider weighted composition operators $W_{\psi, \varphi}: f \mapsto \psi(f \circ \varphi)$ on spaces of analytic functions on the unit disc, which take values in some complex Banach space. We provide necessary and sufficient conditions for the boundedness and (weak) compactness of $W_{\psi, \varphi}$ on general function spaces, and in particular on weak vector-valued spaces. As an application, we characterize the weak compactness of $W_{\psi, \varphi}$ between two different vector-valued Bloch-type spaces. This characterization appears to be new also in the scalar-valued case.
\end{abstract}

Keywords: Bloch-type space, compactness, composition operator, weak compactness, vector-valued function

MSC 2010: Primary 47B38; secondary 46E15, 46E40, 30H30

\section{Introduction}

Let $\mathbb{D}$ be the open unit disc in the complex plane $\mathbb{C}$ and, for any complex Banach space $X$, let $H(\mathbb{D}, X)$ denote the linear space of all analytic functions $f: \mathbb{D} \rightarrow X$. A weighted composition operator $W_{\psi, \varphi}$ defined from $H(\mathbb{D}, X)$ to $H(\mathbb{D}, Y)$ is the linear map

$$
\left(W_{\psi, \varphi} f\right)(z)=\psi(z) f(\varphi(z)), \quad z \in \mathbb{D},
$$

where $\varphi$ is an analytic self-map of the unit disc $\mathbb{D}$ and $\psi \in H(\mathbb{D}, L(X, Y))$. Here $L(X, Y)$ is the space of bounded linear operators from $X$ to $Y$. Weighted composition operators appear naturally; we have $W_{\psi, \varphi}=M_{\psi} C_{\varphi}$, where $M_{\psi}$ is the operator-valued multiplier $f \mapsto \psi f$ and $C_{\varphi}$ is the composition operator $f \mapsto f \circ \varphi$. Moreover, for a large class of Banach spaces $X$, all linear onto isometries between $X$-valued $H^{\infty}$ spaces are of the form (1.1) for suitable $\psi$ and $\varphi$; see $[4,15]$. Properties of multipliers and (weighted) composition operators have been widely studied on various spaces of vector-valued analytic functions, including vector-valued Hardy, Bergman, BMOA and Bloch spaces; see, for example, [2, 3, 10-12, 17-19] and the recent survey [13].

The study of composition operators $C_{\varphi}$ on vector-valued function spaces involves some important basic principles which hold for large classes of function spaces. For example, if $X$ is infinite dimensional, then composition operators $C_{\varphi}$ are not compact on classical $X$-valued analytic function spaces; see, e.g., [13, 17]. On the other hand, for the weak compactness of $C_{\varphi}$ it is necessary that $X$ is reflexive and that $C_{\varphi}$ is weakly compact on the corresponding scalar-valued function space. For the latter statement a partial converse holds for the so-called weak vector-valued function spaces $[2,13]$ as follows: for any Banach space $E$ of analytic

\footnotetext{
Mostafa Hassanlou: Shahid Bakeri High Education Center of Miandoab, Urmia University, Urmia, Iran, e-mail: m.hassanlou@urmia.ac.ir

*Corresponding author: Jussi Laitila: Department of Mathematics and Statistics, University of Helsinki, P.0. Box 68, FI-00014 University of Helsinki, Helsinki, Finland, e-mail: jlaitila@iki.fi

Hamid Vaezi: Department of Pure Mathematics, University of Tabriz, Tabriz, Iran, e-mail: hvaezi@tabrizu.ac.ir
} 
functions $f \in H(\mathbb{D}, \mathbb{C})$ such that $E$ contains the constant functions and the closed unit ball $B_{E}$ is compact in the compact open topology of $\mathbb{D}$, the weak space $w E(X)$ (modelled on $E$ ) is the space of functions $f \in H(\mathbb{D}, X)$ such that

$$
\|f\|_{w E(X)}=\sup _{\left\|x^{*}\right\| X^{*} \leq 1}\left\|x^{*} \circ f\right\|_{E}<\infty .
$$

The first aim of this paper is to present analogous necessary and sufficient conditions for the boundedness and (weak) compactness of weighted composition operators between two different function spaces, where the weight function $\psi$ is scalar valued, i.e., $\psi \in H(\mathbb{D}, \mathbb{C})$. Consequently, we obtain characterizations of the weak compactness of weighted composition operators between many weak vector-valued spaces, such as weak Hardy and Bergman spaces.

In the second part, we characterize the weak compactness of weighted composition operators between two Bloch-type function spaces. For $\alpha \in(0, \infty)$ and a complex Banach space $X$, the vector-valued Bloch-type space $\mathcal{B}_{\alpha}(X)$ is the Banach space of functions $f \in H(\mathbb{D}, X)$ such that

$$
\|f\|_{\mathcal{B}_{\alpha}(X)}=\|f(0)\|_{X}+\sup _{z \in \mathbb{D}}\left\|f^{\prime}(z)\right\|_{X}\left(1-|z|^{2}\right)^{\alpha}<\infty .
$$

In the special case $X=\mathbb{C}$, we get the usual scalar-valued Bloch-type spaces $\mathcal{B}_{\alpha}=\mathcal{B}_{\alpha}(\mathbb{C})$. In the case $\alpha=1$, we get the usual Bloch space $\mathcal{B}(X)=\mathcal{B}_{1}(X)$ (see, for example, [1]). In this part, the main argument concerns a characterization of the weakly compact weighted composition operators between two scalar-valued Blochtype spaces $\mathcal{B}_{\alpha}$ and $\mathcal{B}_{\beta}$. The vector-valued characterization follows from this by applying the general result for weak vector-valued function spaces.

Recall that an operator $T \in L(X, Y)$ is (weakly) compact if for any bounded sequence $\left(x_{n}\right)$ in $X$ there is a (weakly) convergent subsequence $\left(T x_{n_{k}}\right)$ in $Y$. Any compact operator is clearly weakly compact.

\section{Weighted composition operators on general vector-valued function spaces}

In this section we consider weighted composition operators between general spaces of vector-valued analytic functions. Most arguments extend known results for composition operators but we present the details for completeness. Below we assume that the weight function $\psi$ is complex valued $(\psi \in H(\mathbb{D}, \mathbb{C}))$ and nonzero $(\psi(z) \neq 0$ for some $z \in \mathbb{D})$.

Suppose that $A$ is any Banach space of analytic functions $\mathbb{D} \rightarrow \mathbb{C}$ and let $A(X)$ be an associated vectorvalued Banach space of analytic functions $\mathbb{D} \rightarrow X$, where $X$ is a complex Banach space. Assume that the following properties hold for the pair $(A, A(X))$ for all Banach spaces $X$ (see [13, Section 2]):

(a1) The constant maps $f(z) \equiv c$ belong to $A$ for all $c \in \mathbb{C}$.

(a2) $f \mapsto f \otimes x$ defines a bounded linear operator $J_{X}: A \rightarrow A(X)$ for any $x \in X$, where $(f \otimes x)(z)=f(z) x$ for $z \in \mathbb{D}$.

(a3) $f \mapsto x^{*} \circ f$ defines a bounded linear operator $Q_{x^{*}}: A(X) \rightarrow A$ for any $x^{*} \in X^{*}$.

(a4) The point evaluations $\delta_{z}$, where $\delta_{z}(f)=f(z)$ for $f \in A(X)$, are bounded $A(X) \rightarrow X$ for all $z \in \mathbb{D}$.

It follows from these assumptions that the vector-valued constant maps $z \mapsto f_{X}(z)=1 \otimes X \equiv x$ belong to $A(X)$ for all $x \in X$; see [13]. The properties (a1)-(a4) are satisfied by a large number of well-known function spaces, such as $X$-valued Hardy, Bergman, BMOA, and Bloch spaces as well as their weak versions (see, for example, [13] for the definitions of these function spaces).

Suppose next that $A(X)$ and $B(X)$ are two spaces of analytic functions $\mathbb{D} \rightarrow X$ such that the pairs $(A, A(X))$ and $(B, B(X))$ satisfy (a1)-(a4). The following result extends [13, Corollary 2] and [2, Proposition 1] to weighted composition operators. The result involves general operator ideals in the sense of Pietsch [21], although the remainder of the paper mainly concerns the closed ideals of compact and weakly compact operators (but see Remark 2.5 below). For clarity we will use the notation $\widetilde{W}_{\psi, \varphi}$ for the weighted composition operator between vector-valued spaces $A(X) \rightarrow B(X)$ and $W_{\psi, \varphi}$ for the operator between corresponding complex-valued spaces $A \rightarrow B$. We denote by $I_{X}$ the identity operator of $X$. 
Proposition 2.1. Let $X$ be a complex Banach space. Let $\psi \in H(\mathbb{D}, \mathbb{C})$ be nonzero and let $\varphi$ be an analytic selfmap of $\mathbb{D}$. Then we have the following results:

(1) If $\widetilde{W}_{\psi, \varphi}$ is bounded $A(X) \rightarrow B(X)$, then $W_{\psi, \varphi}$ is bounded $A \rightarrow B$.

(2) If $\widetilde{W}_{\psi, \varphi}: A(X) \rightarrow B(X)$ belongs to an operator ideal, then $I_{X}$ and $W_{\psi, \varphi}: A \rightarrow B$ belong to the same operator ideal.

Proof. Let $x \in X, x^{*} \in X^{*}$ be norm-1 vectors such that $\left\langle x^{*}, x\right\rangle=1$, let $z_{0} \in \mathbb{D}$ be a point such that $\psi\left(z_{0}\right) \neq 0$, and let $j_{1}, j_{2}, j_{3}, j_{4}$ be the linear operators

$$
\begin{array}{ll}
j_{1}: A \rightarrow A(X), & f \mapsto f \otimes x, \\
j_{2}: B(X) \rightarrow B, & f \mapsto x^{*} \circ f, \\
j_{3}: X \rightarrow A(X), & x \mapsto f_{x}, \\
j_{4}: B(X) \rightarrow X, & f \mapsto f\left(z_{0}\right) / \psi\left(z_{0}\right) .
\end{array}
$$

By (a1)-(a4), all these operators are bounded, and

$$
W_{\psi, \varphi}=j_{2} \circ \widetilde{W}_{\psi, \varphi} \circ j_{1}, \quad I_{X}=j_{4} \circ \widetilde{W}_{\psi, \varphi} \circ j_{3} .
$$

The following result settles the question of compactness of $\widetilde{W}_{\psi, \varphi}$ between vector-valued spaces satisfying (a1)-(a4).

Proposition 2.2. Let $\psi \in H(\mathbb{D}, \mathbb{C})$ be nonzero and let $\varphi$ be an analytic self-map of $\mathbb{D}$. Then $\widetilde{W}_{\psi, \varphi}$ is compact $A(X) \rightarrow B(X)$ if and only if $X$ is finite dimensional and $W_{\psi, \varphi}$ is compact $A \rightarrow B$.

Proof. Applied to the closed operator ideal of compact operators, Proposition 2.1 implies that if $\widetilde{W}_{\psi, \varphi}$ is compact, then $X$ is finite dimensional and $W_{\psi, \varphi}$ is compact. The proof of the converse direction is a straightforward modification of the proof of [13, Proposition 7] and is thus omitted.

Let us next consider the general class of weak vector-valued function spaces (1.2), introduced by Bonet, Domański and Lindström [2] in the context of composition operators. Suppose that $E$ is a Banach space of analytic functions $f \in H(\mathbb{D}, \mathbb{C})$ satisfying the following conditions:

(b1) $E$ contains the constant functions,

(b2) the closed unit ball $B_{E}$ is compact in the compact open topology $\tau_{\text {co }}$ of $\mathbb{D}$.

Then the weak space defined in (1.2) is a Banach space; see [2]. Moreover, by [13, Lemma 13], properties (b1) and (b2) imply the assumptions (a1)-(a4), so that Propositions 2.1 and 2.2 apply to weak spaces.

The next result provides converses to Proposition 2.1 for bounded and weakly compact weighted composition operators between two different weak spaces. It also extends [2, Proposition 11] to weighted composition operators. Recall here that the identity operator $I_{X}$ of $X$ is weakly compact if and only if $X$ is reflexive.

Proposition 2.3. Let $\psi \in H(\mathbb{D}, \mathbb{C})$ and let $\varphi$ be an analytic self-map of $\mathbb{D}$. Suppose that $E_{1}$ and $E_{2}$ are spaces of analytic functions on $\mathbb{D}$ that satisfy assumptions (b1) and (b2). Then we have the following results:

(1) If $W_{\psi, \varphi}: E_{1} \rightarrow E_{2}$ is bounded, then $\widetilde{W}_{\psi, \varphi}: w E_{1}(X) \rightarrow w E_{2}(X)$ is bounded.

(2) If $X$ is a reflexive Banach space and $W_{\psi, \varphi}: E_{1} \rightarrow E_{2}$ is compact, then the operator $\widetilde{W}_{\psi, \varphi}$ is weakly compact $w E_{1}(X) \rightarrow w E_{2}(X)$.

For the proof of Proposition 2.3 we need the following facts from [2]: Assumptions (b1) and (b2) imply that the point evaluation maps $\delta_{z}$ belong to $E^{*}$ for each $z \in \mathbb{D}$ and that $E$ is the dual space of $V_{E}$, where $V_{E}$ is the closed linear span in $E^{*}$ of $\left\{\delta_{z} \in E^{*}: z \in \mathbb{D}\right\}$. Moreover, by [2, Lemma 10], there is an isometric isomorphism $\chi: L\left(V_{E}, X\right) \rightarrow w E(X)$ so that

$$
(\chi(T))(z)=T\left(\delta_{z}\right) \quad \text { and } \quad\left(\chi^{-1}(f)\right)\left(\delta_{z}\right)=f(z)
$$

hold for $T \in L\left(V_{E}, X\right), f \in w E(X)$ and $z \in \mathbb{D}$.

Proof of Proposition 2.3. For part (1) note that

$$
\left\|x^{*} \circ\left(\widetilde{W}_{\psi, \varphi} f\right)\right\|_{E_{2}}=\left\|W_{\psi, \varphi}\left(x^{*} \circ f\right)\right\|_{E_{2}} \leq\left\|W_{\psi, \varphi}\right\| \cdot\left\|x^{*} \circ f\right\|_{E_{1}}
$$

so that $\left\|\widetilde{W}_{\psi, \varphi}\right\| \leq\left\|W_{\psi, \varphi}\right\|$. 
We next prove part (2). Assume that $W_{\psi, \varphi}: E_{1} \rightarrow E_{2}$ is compact. Its adjoint $\left(W_{\psi, \varphi}\right)^{*}: E_{2}^{*} \rightarrow E_{1}^{*}$ satisfies

$$
\left(W_{\psi, \varphi}\right)^{*}\left(\delta_{z}\right)=\psi(z) \delta_{\varphi(z)}, \quad z \in \mathbb{D}
$$

so that $\left(W_{\psi, \varphi}\right)^{*}\left(V_{E_{2}}\right) \subset V_{E_{1}}$. We obtain the factorization $\widetilde{W}_{\psi, \varphi}=\chi_{2} \circ S_{\psi, \varphi} \circ \chi_{1}^{-1}$, where $S_{\psi, \varphi}$ is the operator composition map

$$
\left.T \mapsto I_{L\left(V_{E_{1}}, X\right)} \circ T \circ\left(W_{\psi, \varphi}\right)^{*}\right|_{V_{E_{2}}}, \quad L\left(V_{E_{2}}, X\right) \rightarrow L\left(V_{E_{1}}, X\right),
$$

and $\chi_{1}: L\left(V_{E_{1}}, X\right) \rightarrow w E_{1}(X)$ and $\chi_{2}: L\left(V_{E_{2}}, X\right) \rightarrow w E_{2}(X)$ are isometric isomorphisms from (2.1). Indeed,

$$
\begin{aligned}
\left(\left(\chi_{2} \circ S_{\psi, \varphi} \circ \chi_{1}^{-1}\right)(f)\right)(z) & =\left(\chi_{2}\left(\left.\chi_{1}^{-1}(f) \circ\left(W_{\psi, \varphi}\right)^{*}\right|_{V_{E_{2}}}\right)\right)(z)=\chi_{1}^{-1}(f)\left(\left(W_{\psi, \varphi}\right)^{*}\left(\delta_{z}\right)\right) \\
& =\chi_{1}^{-1}(f)\left(\psi(z) \delta_{\varphi(z)}\right)=\psi(z) \chi_{1}^{-1}(f)\left(\delta_{\varphi(z)}\right)=\psi(z)(f(\varphi(z))
\end{aligned}
$$

for $f \in E_{1}$ and $z \in \mathbb{D}$.

Since $\left.\left(W_{\psi, \varphi}\right)^{*}\right|_{V_{E_{2}}}$ is a compact operator $V_{E_{2}} \rightarrow V_{E_{1}}$ by duality and $I_{X}$ is weakly compact by reflexivity of $X$, it follows from [22, Proposition 2.3 and Remark 2.4], that the operator composition $S_{\psi, \varphi}$ is weakly compact $L\left(V_{E_{2}}, X\right) \rightarrow L\left(V_{E_{1}}, X\right)$. Consequently, $\widetilde{W}_{\psi, \varphi}$ is weakly compact $w E_{1}(X) \rightarrow w E_{2}(X)$.

As concrete examples, let us next consider weak versions of two classical function spaces: the Hardy space $H^{1}$ and the Bergman space $A^{1}$. Recall that $f \in H(\mathbb{D}, \mathbb{C})$ belongs to $H^{1}$ if and only if

$$
\|f\|_{H^{1}}=\sup _{0<r<1} \frac{1}{2 \pi} \int_{0}^{2 \pi}\left|f\left(r \mathrm{e}^{i \theta}\right)\right| \mathrm{d} \theta<\infty,
$$

and $f \in H(\mathbb{D}, \mathbb{C})$ belongs to $A^{1}$ if and only if

$$
\|f\|_{A^{1}}=\int_{\mathbb{D}}|f(z)| \mathrm{d} A(z)<\infty,
$$

where $A$ is the normalized area measure on $\mathbb{D}$. Both of these spaces satisfy (b1) and (b2), so the associated weak spaces $w H^{1}(X)$ and $w A^{1}(X)$ are Banach spaces for all $X$. However, they are quite different from the usual “strong” spaces $H^{1}(X)$ and $A^{1}(X)$; see $[11,14]$.

Compactness and weak compactness of $W_{\psi, \varphi}$ are well understood on $H^{1}$ and $A^{1}$; function-theoretic characterizations exist for the compactness of $W_{\psi, \varphi}$ on both $H^{1}$ and $A^{1}$; see [7,8]. In [7] it was further shown that every weakly compact $W_{\psi, \varphi}$ on $H^{1}$ is compact. Moreover, because $A^{1}$ is isomorphic to $\ell^{1}$, it has the Schur property, and so every weakly compact linear operator on $A^{1}$ is compact; see [17, p. 302]. Using Proposition 2.3, we get the following extension of these scalar results.

Corollary 2.4. Let $\psi \in H(\mathbb{D}, \mathbb{C})$ be nonzero and let $\varphi$ be an analytic self-map of $\mathbb{D}$. Then we have the following: (1) $\widetilde{W}_{\psi, \varphi}$ is weakly compact on $w H^{1}(X)$ if and only if $X$ is reflexive and $W_{\psi, \varphi}$ is compact on $H^{1}$.

(2) $\widetilde{W}_{\psi, \varphi}$ is weakly compact on $w A^{1}(X)$ if and only if $X$ is reflexive and $W_{\psi, \varphi}$ is compact on $A^{1}$.

Corollary 2.4 could include more examples. For example, (weak) compactness of $W_{\psi, \varphi}$ between weighted spaces $H_{v}^{\infty}$ is well known $[5,6]$. Also the weak space $w H_{v}^{\infty}(X)$ is well defined (and coincides with the usual “strong” space $\left.H_{v}^{\infty}(X)\right)$. However, weak compactness of the more general "operator-weighted" compositions $\widetilde{W}_{\psi, \varphi}\left(\right.$ where $\psi \in H(\mathbb{D}, L(X, Y))$ ) between $H_{v}^{\infty}(X)$ spaces has been characterized in [12].

It is worthwhile to note that the general framework (Propositions 2.1, 2.2 and 2.3) does not in general hold for operator-weighted composition operators. For example, an operator-weighted composition between weighted $H^{\infty}(X)$ spaces can be compact even if $X$ is infinite dimensional [12]. Indeed, the present knowledge of operator-valued composition operators is quite rudimentary and, for example, their boundedness and compactness on Hardy spaces $H^{p}(X)$ is still an open question; see the discussion in [13, Section 7].

Remark 2.5. An analogue of Proposition 2.3 (2) holds for the ideal of weakly conditionally compact operators: Recall that an operator $T \in L(X, Y)$ is weakly conditionally compact if for any bounded sequence $\left(x_{n}\right)$ in $X$ there is a weakly Cauchy subsequence $\left(T x_{n_{k}}\right)$ in $Y$. Then, if $X$ does not contain an isomorphic copy of $\ell^{1}$ and $W_{\psi, \varphi}: E_{1} \rightarrow E_{2}$ is compact, it follows that $\widetilde{W}_{\psi, \varphi}$ is weakly conditionally compact $w E_{1}(X) \rightarrow w E_{2}(X)$. The proof is analogous to Proposition 2.3 (2), but uses [16] instead to deduce that the operator composition $S_{\psi, \varphi}$ is weakly conditionally compact. 


\section{Weighted composition operators between Bloch-type spaces}

We next consider weighted composition operators between Bloch-type spaces $\mathcal{B}_{\alpha}(X)$. Boundedness and compactness of weighted composition operators between two scalar-valued Bloch-type spaces $\mathcal{B}_{\alpha}$ is well known. Ohno, Stroethoff and Zhao [20] provided the following function-theoretic characterizations, which involve the quantities

$$
\begin{aligned}
q_{1}(\alpha, \beta, z) & =\frac{\left(1-|z|^{2}\right)^{\beta}}{\left(1-|\varphi(z)|^{2}\right)^{\alpha}}|\psi(z)|\left|\varphi^{\prime}(z)\right|, \\
q_{2}(\beta, z) & =\left(1-|z|^{2}\right)^{\beta} \log \frac{1}{1-|\varphi(z)|^{2}}\left|\psi^{\prime}(z)\right|, \\
q_{3}(\alpha, \beta, z) & =\frac{\left(1-|z|^{2}\right)^{\beta}}{\left(1-|\varphi(z)|^{2}\right)^{\alpha-1}}\left|\psi^{\prime}(z)\right|
\end{aligned}
$$

for $\alpha, \beta \in(0, \infty)$ :

(1) If $0<\alpha<1$, then $W_{\psi, \varphi}: \mathcal{B}_{\alpha} \rightarrow \mathcal{B}_{\beta}$ is bounded or compact if and only if

$$
\sup _{z \in \mathbb{D}} q_{1}(\alpha, \beta, z)<\infty \quad \text { or } \quad \lim _{|\varphi(z)| \rightarrow 1} q_{1}(\alpha, \beta, z)=0,
$$

respectively.

(2) If $\alpha=1$, then $W_{\psi, \varphi}: \mathcal{B} \rightarrow \mathcal{B}_{\beta}$ is bounded or compact if and only if

$$
\sup _{z \in \mathbb{D}} q_{1}(1, \beta, z)<\infty \text { and } \sup _{z \in \mathbb{D}} q_{2}(\beta, z)<\infty,
$$

or

$$
\lim _{|\varphi(z)| \rightarrow 1} q_{1}(1, \beta, z)=0 \quad \text { and } \quad \lim _{|\varphi(z)| \rightarrow 1} q_{2}(\beta, z)=0
$$

respectively.

(3) If $1<\alpha<\infty$, then $W_{\psi, \varphi}: \mathcal{B}_{\alpha} \rightarrow \mathcal{B}_{\beta}$ is bounded or compact if and only if

$$
\sup _{z \in \mathbb{D}} q_{1}(\alpha, \beta, z)<\infty \text { and } \sup _{z \in \mathbb{D}} q_{3}(\alpha, \beta, z)<\infty,
$$

or

$$
\lim _{|\varphi(z)| \rightarrow 1} q_{1}(\alpha, \beta, z)=0 \quad \text { and } \quad \lim _{|\varphi(z)| \rightarrow 1} q_{3}(\alpha, \beta, z)=0
$$

respectively.

The following theorem extends the scalar results to vector-valued Bloch-type spaces. For composition operators, the case $\alpha=\beta=1$ is from [17, Theorem 4] and the case $\alpha=\beta \in(0, \infty)$ follows from [2, Proposition 11]. Our proof applies an approach similar to that of [17].

Theorem 3.1. Let $\psi \in H(\mathbb{D}, \mathbb{C})$ be nonzero and let $\varphi$ be an analytic self-map of $\mathbb{D}$. Let $\alpha, \beta \in(0, \infty)$. Let $X$ be a complex Banach space.

(1) $W_{\psi, \varphi}$ is weakly compact $\mathcal{B}_{\alpha} \rightarrow \mathcal{B}_{\beta}$ if and only if it is compact.

(2) $\widetilde{W}_{\psi, \varphi}$ is bounded $\mathcal{B}_{\alpha}(X) \rightarrow \mathcal{B}_{\beta}(X)$ if and only if $W_{\psi, \varphi}$ is bounded $\mathcal{B}_{\alpha} \rightarrow \mathcal{B}_{\beta}$.

(3) $\widetilde{W}_{\psi, \varphi}$ is weakly compact $\mathcal{B}_{\alpha}(X) \rightarrow \mathcal{B}_{\beta}(X)$ if and only if $W_{\psi, \varphi}$ is (weakly) compact $\mathcal{B}_{\alpha} \rightarrow \mathcal{B}_{\beta}$ and $X$ is reflexive.

For the proof of Theorem 3.1, we will need the following facts: For $\alpha \in(0, \infty)$ let $\mathcal{B}_{\alpha, 0}$ denote the little Blochtype space, which is the closed subspace of $\mathcal{B}_{\alpha}$ for which

$$
\lim _{|z| \rightarrow 1}\left|f^{\prime}(z)\right|\left(1-|z|^{2}\right)^{\alpha}=0 .
$$

First, by [23, Theorem 15], the Bergman space $A^{1}$ is the dual space of $\mathcal{B}_{\alpha, 0}$ for all $\alpha$ under the pairing

$$
\langle f, g\rangle_{\alpha}=\lim _{r \rightarrow 1} \int_{\mathbb{D}} f(r z) \overline{g(r z)}\left(1-|z|^{2}\right)^{\alpha-1} \mathrm{~d} A(z), \quad f \in \mathcal{B}_{\alpha, 0}, g \in A^{1}
$$


where $\mathrm{d} A(z)$ is the normalized area measure on $\mathbb{D}$. Second, each $\mathcal{B}_{\alpha, 0}$ has the Dunford-Pettis property (DPP). Recall that a Banach space $E$ has the DPP whenever every weakly compact operator from $E$ to some Banach space is completely continuous, i.e., if it maps weakly null sequences to norm null sequences. The fact that $\mathcal{B}_{\alpha, 0}$ has the DPP follows from the above duality and the fact that $A^{1} \sim \ell^{1}$ has the Schur property; see, for example, $[9,17]$.

Proof of Theorem 3.1. It is easy to see that each $\mathcal{B}_{\alpha}(X)$ is a weak space of the form (1.2). In fact, we have $\mathcal{B}_{\alpha}(X)=w \mathcal{B}_{\alpha}(X)$ with equivalent norms, where $w \mathcal{B}_{\alpha}(X)$ is modelled on $\mathcal{B}_{\alpha}$. Therefore, parts (2) and (3) of Theorem 3.1 follow from part (1) and Propositions 2.1 and 2.3. We thus only need to prove part (1).

Let first $\alpha, \beta \in(0, \infty)$ and suppose that $W_{\psi, \varphi}$ is weakly compact, but not compact, $\mathcal{B}_{\alpha} \rightarrow \mathcal{B}_{\beta}$. Then there are $\varepsilon>0$ and a sequence $\left(z_{n}\right) \subset \mathbb{D}$ so that $\left|\varphi\left(z_{n}\right)\right| \rightarrow 1$ and $q_{1}\left(\alpha, \beta, z_{n}\right) \geq \varepsilon$ for all $n$. For $n \geq 0$ define the functions $f_{n} \in H(\mathbb{D}, \mathbb{C})$ by

$$
f_{n}(z)=\frac{\left(1-\left|\varphi\left(z_{n}\right)\right|^{2}\right)^{2}}{\left(1-\overline{\varphi\left(z_{n}\right)} z\right)^{\alpha+1}}-\frac{1-\left|\varphi\left(z_{n}\right)\right|^{2}}{\left(1-\overline{\varphi\left(z_{n}\right)} z\right)^{\alpha}}
$$

for $z \in \mathbb{D}$. In [20, p. 202] it has been shown that the sequence $\left(f_{n}\right)$ is uniformly bounded in $\mathcal{B}_{\alpha}, f_{n}(z) \rightarrow 0$ uniformly on compact subsets of $\mathbb{D}, f_{n}\left(\varphi\left(z_{n}\right)\right)=0$, and $f_{n}^{\prime}\left(\varphi\left(z_{n}\right)\right)=\overline{\varphi\left(z_{n}\right)} /\left(1-\left|\varphi\left(z_{n}\right)\right|^{2}\right)^{\alpha}$. Thus,

$$
\left\|W_{\psi, \varphi} f_{n}\right\|_{\mathcal{B}_{\beta}} \geq\left|\left(W_{\psi, \varphi} f_{n}\right)^{\prime}\left(z_{n}\right)\right|\left(1-\left|z_{n}\right|\right)^{\beta}=\left|\varphi\left(z_{n}\right)\right| q_{1}\left(\alpha, \beta, z_{n}\right) \geq \frac{\varepsilon}{2}
$$

for all $n$ large enough. We further have $f_{n} \in \mathcal{B}_{\alpha, 0}$ for all $n$. Let $\widehat{f}_{n}(j)$ denote the $j$ th Taylor coefficient of $f_{n}$ and let $p_{N}(z)=\sum_{j=0}^{N} a_{j} z^{j}$ be a polynomial. Since $f_{n}(z) \rightarrow 0$ uniformly on compact subsets of $\mathbb{D}$, we have

$$
\widehat{f}_{n}(j)=\frac{f_{n}^{(j)}(0)}{j !} \rightarrow 0 \quad \text { as } \quad n \rightarrow \infty
$$

By (3.1), we have

$$
\begin{aligned}
\left\langle f_{n}, p_{N}\right\rangle_{\alpha} & =\lim _{r \rightarrow 1} \int_{0}^{2 \pi} \int_{0}^{1} \sum_{k=0}^{\infty} \sum_{j=0}^{N} \widehat{f}_{n}(k) \overline{a_{j}} r^{k+j} s^{k+j+1} \mathrm{e}^{i(k-j) \theta}\left(1-s^{2}\right)^{\alpha-1} \mathrm{~d} s \frac{\mathrm{d} \theta}{2 \pi} \\
& =\sum_{j=0}^{N} \widehat{f}_{n}(j) \overline{a_{j}} \int_{0}^{1} s^{2 j+1}\left(1-s^{2}\right)^{\alpha-1} \mathrm{~d} s \rightarrow 0
\end{aligned}
$$

as $n \rightarrow \infty$. Since the polynomials are dense in $A^{1}$, this means that $f_{n} \rightarrow 0$ weakly in $\mathcal{B}_{\alpha, 0}$. Weak compactness of $W_{\psi, \varphi}$ and the DPP of $\mathcal{B}_{\alpha, 0}$ now imply that $W_{\psi, \varphi} f_{n} \rightarrow 0$ in $\mathcal{B}_{\beta, 0}$ and thus in $\mathcal{B}_{\beta}$. This contradicts (3.2).

We next divide the proof into the following three cases: (i) $\alpha \in(0,1)$, (ii) $\alpha=1$, and (iii) $\alpha \in(1, \infty)$. The above argument proves case (i). Cases (ii) and (iii) are completed similarly, by using functions

$$
g_{n}(z)=\frac{-1}{\log \left(1-\left|\varphi\left(z_{n}\right)\right|^{2}\right)}\left(3\left(\log \frac{1}{1-\overline{\varphi\left(z_{n}\right)} z}\right)^{2}-2\left(\log \frac{1}{1-\overline{\varphi\left(z_{n}\right)} z}\right)^{3}\right)
$$

or

$$
h_{n}(z)=(\alpha+1) \frac{1-\left|\varphi\left(z_{n}\right)\right|^{2}}{\left(1-\overline{\varphi\left(z_{n}\right)} z\right)^{\alpha}}-\alpha \frac{\left(1-\left|\varphi\left(z_{n}\right)\right|^{2}\right)^{2}}{\left(1-\overline{\varphi\left(z_{n}\right)} z\right)^{\alpha+1}},
$$

respectively, instead of $f_{n}$.

Acknowledgment: We thank Hans-Olav Tylli for his comments on a preliminary version of the manuscript. 


\section{References}

[1] J. Arregui and O. Blasco, Bergman and Bloch spaces of vector-valued functions, Math. Nachr. 261-262 (2003), 3-22.

[2] J. Bonet, P. Domański and M. Lindström, Weakly compact composition operators on analytic vector-valued function spaces, Ann. Acad. Sci. Fenn. Math. 26 (2001), 233-248.

[3] J. Bonet, M. C. Gómez-Collado, D. Jornet and E. Wolf, Operator-weighted composition operators between weighted spaces of vector-valued analytic functions, Ann. Acad. Sci. Fenn. Math. 37 (2012), 319-338.

[4] M. Cambern and K. Jarosz, Multipliers and isometries in $H_{E}^{\infty}$, Bull. Lond. Math. Soc. 22 (1990), 463-466.

[5] M. D. Contreras and S. Díaz-Madrigal, Compact-type operators defined on $H^{\infty}$, Contemp. Math. 232 (1999), 111-118.

[6] M. D. Contreras and A. G. Hernández-Díaz, Weighted composition operators in weighted Banach spaces of analytic functions, J. Aust. Math. Soc. 69 (2000), 41-60.

[7] M. D. Contreras and A. G. Hernández-Díaz, Weighted composition operators on Hardy spaces, J. Math. Anal. Appl. 263 (2001), 224-233.

[8] Ž. Čučković and R. Zhao, Weighted composition operators on the Bergman space, J. Lond. Math. Soc. (2) 70 (2004), 499-511.

[9] J. Diestel, A survey of results related to the Dunford-Pettis property, Contemp. Math. 2 (1980), 15-60.

[10] M. Hassanlou, H. Vaezi and M. Wang, Weighted composition operators on weak vector-valued weighted Bergman spaces and Hardy spaces, Banach J. Math. Anal. 9 (2015), 35-43.

[11] J. Laitila and H.-O. Tylli, Composition operators on vector-valued harmonic functions and Cauchy transforms, Indiana Univ. Math. J. 55 (2006), 719-746.

[12] J. Laitila and H.-O. Tylli, Operator-weighted composition operators on vector-valued analytic function spaces, Illinois J. Math. 53 (2009), 1019-1032.

[13] J. Laitila and H.-O. Tylli, Composition operators on vector-valued analytic function spaces: A survey, Acta Comment. Univ. Tartu. Math. 18 (2014), 135-157.

[14] J. Laitila, H.-O. Tylli and M. Wang, Composition operators from weak to strong spaces of vector-valued analytic functions, J. Operator Theory 62 (2009), 281-295.

[15] P.-K. Lin, The isometries of $H^{\infty}($ E), Pacific J. Math. 143 (1990), 69-77.

[16] M. Lindström and G. Schlüchtermann, Composition of operator ideals, Math. Scand. 84 (1999), 284-296.

[17] P. Liu, E. Saksman and H.-O. Tylli, Small composition operators on analytic vector-valued function spaces, Pacific J. Math. 184 (1998), 295-309.

[18] J. S. Manhas, Multiplication operators on weighted locally convex spaces of vector-valued analytic functions, Southeast Asian Bull. Math. 27 (2003), 649-660.

[19] J. S. Manhas, Weighted composition operators on weighted spaces of vector-valued analytic functions, J. Korean Math. Soc. 45 (2008), 1203-1220.

[20] S. Ohno, K. Stroethoff and R. Zhao, Weighted composition operators between Bloch-type spaces, Rocky Mountain J. Math. 33 (2003), 191-215.

[21] A. Pietsch, Operator Ideals, North-Holland, Amsterdam, 1980.

[22] É. Saksman and H.-O. Tylli, Multiplications and elementary operators in the Banach space setting, in: Methods in Banach Space Theory, London Math. Soc. Lecture Note Ser. 337, Cambridge University Press, Cambridge (2006), 253-292.

[23] K. Zhu, Bloch type spaces of analytic functions, Rocky Mountain J. Math. 23 (1993), 1143-1177. 